\title{
Reply to Comments on 'Gait Disturbance due to Foot Drop Is Refractory to Treatment in Nonsystemic Vasculitic Neuropathy'
}

\author{
Tomoo Hirahara $^{a}$ Satoshi Yamashita ${ }^{a}$ Yohei Misumi ${ }^{\mathrm{a}}$ Kensuke Kawakami ${ }^{\mathrm{a}}$ \\ Hiroko Hori $^{\mathrm{a}}$ Shoji Honda ${ }^{\mathrm{a}}$ Masaki Watanabe ${ }^{\mathrm{a}}$ Tokunori lkeda ${ }^{\mathrm{a}} \mathrm{b}$ \\ Taro Yamashita $^{a}$ Yasushi Maeda ${ }^{a}$ Yukio Ando ${ }^{a}$ \\ Departments of a Neurology and ${ }^{b}$ Immunogenetics, Graduate School of Medical Sciences, \\ Kumamoto University, Kumamoto, Japan
}

Dear Sir,

We welcome the comments of Drs. Collins and Hadden and appreciate their interest in our study regarding nonsystemic vasculitic neuropathy (NSVN). They ask essential and thoughtful inquiries that would clarify the points at issue in our study. We recognize that there are some shortcomings and limitations in some aspects. We provide further clarifications of the eight issues raised by Drs. Collins and Hadden.

First, we differentiate the 'polyneuropathy' and 'mononeuritis multiplex' based on the clinical manifestations: diffuse neuropathies with symmetric features were classified as polyneuropathy, while asymmetric neuropathies that showed an obvious laterality were categorized into mononeuritis multiplex. Second, all the patients in the study complained of severe pain that was resistant to non-steroidal anti-inflammatory drugs. Third, no patients presented extremely delayed motor conduction velocities and distal motor latencies in the study. However, F waves were not evoked in most patients, particularly in patients with extremely low amplitudes in CMAP.
Fourth, two patients (cases 11 and 12) initially presented skin eruptions and consulted primary physicians. However, skin vasculitis was not confirmed pathologically on the biopsied specimens, and then we included them into the study. It might have been inappropriate to include them in the study. Fifth, as far as we examined case 13 systemically, we found no evidence of systemic vasculitis. It might have been inappropriate to include them in the study, according to the diagnostic criteria for vasculitic neuropathy and NSVN. Sixth, for the patients with definite vasculitis, we measured the size of most severely affected vessels with perivascular inflammation. However, in these vessels, it can be difficult to isolate vessel walls from inflammatory infiltrates, and then we possibly overestimated the diameters of affected vessels. For the patients with probable vasculitis, we estimated the size of vessels adjacent to the inflammatory infiltrates in the biopsy specimens. These are presumably reasons why our estimation of size of affected vessels was inconsistent with the previous lit- erature. Seventh, the duration between the initiation of treatment and time when recovery reached a plateau varied among the patients, and ranged from 4 months to 8 years. We tabulated the mRS scores at the time of discharge in table 1 and the time when recovery reached a plateau in table 2 . In this study, some patients disagreed with more intensive therapy including cyclophosphamide presumably because NSVN is unlikely to be a life-threatening disease. We accept the criticism that the prognosis of this cohort might have been worse than that in other studies due to under treatment with cyclophosphamide. However, most patients in the study seemed to be satisfied with independent gait. Therefore, a further study regarding the relationship between the treatment and patient's satisfaction might be useful. Eighth, six of seven patients presented bilateral foot drop. These patients needed short leg braces or canes to walk. Therefore, we still insist that a foot drop predicts to have a worse outcome at least under our therapeutic condition.

\section{KARGER 125}

(c) 2014 S. Karger AG, Base

$0014-3022 / 14 / 0732-0080 \$ 39.50 / 0$
Satoshi Yamashita, MD, $\mathrm{PhD}$

Department of Neurology, Graduate School of Medical Sciences

Kumamoto University, 1-1-1 Honjo

Kumamoto 860-8556 (Japan)

E-Mail y-stsh@ kumamoto-u.ac.jp 